\title{
3D Coronal magnetic field from vector magnetograms: non-constant- $\alpha$ force-free configuration of the active region NOAA 8151
}

\author{
S. Régnier ${ }^{1,3}$, T. Amari ${ }^{2}$, and E. Kersalé ${ }^{2}$ \\ ${ }^{1}$ Institut d'Astrophysique Spatiale, Unité Mixte CNRS-Université Paris XI, Bât. 121, 91405 Orsay, France \\ 2 Centre de Physique Théorique, École Polytechnique, 91128 Palaiseau, France \\ 3 Montana State University, Department of Physics, Bozeman, MT 59717, USA
}

Received 16 May 2002 / Accepted 3 July 2002

\begin{abstract}
The Active Region 8151 (AR 8151) observed in February 1998 is the site of an eruptive event associated with a filament and a S-shaped structure, and producing a slow Coronal Mass Ejection (CME). In order to determine how the CME occurs, we compute the 3D coronal magnetic field and we derive some relevant parameters such as the free magnetic energy and the relative magnetic helicity. The 3D magnetic configuration is reconstructed from photospheric magnetic magnetograms (IVM, Mees Solar Observatory) in the case of a non-constant- $\alpha$ force-free (nlff) field model. The reconstruction method is divided into three main steps: the analysis of vector magnetograms (transverse fields, vertical density of electric current, ambiguity of $180^{\circ}$ ), the numerical scheme for the nlff magnetic field, the interpretation of the computed magnetic field with respect to the observations. For AR 8151, the nlff field matches the coronal observations from EIT/SOHO and from SXT/Yohkoh. In particular, three characteristic flux tubes are shown: a highly twisted flux tube, a long twisted flux tube and a quasi-potential flux tube. The maximum energy budget is estimated to $2.6 \times 10^{31} \mathrm{erg}$ and the relative magnetic helicity to $4.7 \times 10^{34} \mathrm{G}^{2} \mathrm{~cm}^{4}$. From the simple photospheric magnetic distribution and the evidence of highly twisted flux tubes, we argue that the flux rope model is the most likely to describe the initiation mechanism of the eruptive event associated with AR 8151 .
\end{abstract}

Key words. Sun: corona - Sun: magnetic fields - Sun: coronal mass ejections (CMEs)

\section{Introduction}

In the solar atmosphere, eruptive events as flares, coronal mass ejections (CMEs) and filament eruptions are frequently observed. To understand the origin of these phenomena, it is important to know what are the structures involved in those events (e.g. filaments, sigmoids) and what is the evolution in time before the eruption (e.g. energy storage, emergence of flux). As the solar corona is dominated by the magnetic field (i.e., a low $\beta$ plasma), the knowledge of the coronal magnetic field configuration will be able to answer these questions. Unfortunately measurements of the magnetic field in the corona are not yet easily performed in spite of the recent works using the Hanle effect (Raouafi 2000), EUV and radio measurements (e.g. Brosius et al. 1992) or Zeeman splitting from infrared lines (Lin et al. 2000). Therefore methods to determine the topology and the geometry of the coronal magnetic field have been developed: the determination of the geometry of active region loops (height, inclination, radius, width) applying a dynamical stereoscopy method to coronal EUV observations (Aschwanden et al. 1999a, 1999b, 2000), the reconstruction of

Send offprint requests to: S. Régnier,

e-mail: stephane.regnier@ias.u-psud.fr the coronal magnetic field using photospheric measurements as boundary condition (see reviews by Sakurai 1989; Amari \& Démoulin 1992).

The latter method, the so-called reconstruction problem (Amari et al. 1997; McClymont et al. 1997 and reference therein), consists of solving the magnetohydrostatic equations with appropriated boundary conditions. As the solar corona is considered as a low $\beta$ plasma (e.g. Priest 1984), the magnetohydrostatic equilibria can be reduced to three different equilibium states (Sakurai 1989): the current-free field, the constant- $\alpha$ force-free (lff) field and the non-constant- $\alpha$ forcefree (nlff) field. The potential field (no electric current within the magnetic configurations) and the lff field (the electric current proportional to the magnetic field) have been well studied (see review by Sakurai 1989). Using either the longitudinal or the vertical component of the photospheric magnetic field as boundary condition, these methods have been compared to chromospheric and coronal observations (e.g. Mandrini et al. 1997) for active regions with a slow evolution and a relatively simple topology. To determine the 3D magnetic configuration of active regions with a complex topology, the nlff field which takes into account the existence of localized electric currents is more suitable (Démoulin et al. 1997). Due to the 
intrinsic nonlinearity of the nlff field equations, the reconstruction methods are essentially computational methods. A variety of computational methods has been developed for which the main differences are the numerical schemes (Amari et al. 1997) and the boundary conditions imposed at the photospheric level (Amari \& Démoulin 1992; Démoulin et al. 1997). In the present paper, we apply the vector potential Grad-Rubin-like method (Amari et al. 1997; Amari et al. 1999) using the vertical component of the magnetic field, $B_{z}$, and the vertical component of the electric current density, $J_{z}$, for a given magnetic polarity $\left(B_{z}>0\right.$ or $\left.B_{z}<0\right)$ as the boundary condition at the photospheric level (Sakurai 1981). These two vertical components are provided by vector magnetograms.

The paper is organized as follows. In Sect. 2, we describe the characteristic features of the Active Region 8151 (AR 8151) which have motivated its choice for this study. In Sect. 3, we detail the vector magnetic field observations from IVM (Imaging Vector Magnetogram, Mees Solar Observatory, Hawaii). In particular, we analyse the vertical current density $J_{z}$ and the distribution of $\alpha$ on the photosphere. The vector potential Grad-Rubin-like method is described in Sect. 4. The photospheric boundary condition determined in Sect. 3 is used to compute the 3D nlff magnetic field (Sect. 5). We focus on three characteristic flux tubes which are compared to coronal EUV and soft X-ray observations. In Sect. 6, we discuss the implications of the computed nlff magnetic configuration in the CME mechanisms.

\section{Description of the active region}

In most eruptive events (flares, filament eruptions or CMEs), the observed active regions have characteristic magnetic features which take an active or inactive part in the phenomena. Two features are commonly associated with eruptive events: filaments-prominences and/or sigmoids. Using LASCO/SOHO and EIT/SOHO data, Subramanian and Dere (2001) have shown that $44 \%$ of the 32 studied CMEs are related to filament eruptions occuring in active regions. From X-ray images (SXT/Yohkoh), Canfield et al. (2000) have deduced that the existence of a sigmoid (S-shaped or reverse S-shaped structure) in active regions is a precursor of eruptive events. These two structures are observed in AR 8151. In addition AR 8151 has a relatively simple distribution of the photospheric magnetic field as we show in the following description.

AR 8151 was observed on February 5-14, 1998 during Carrington Rotation 1932. AR 8151 is located in the South hemisphere with an average latitude of $24^{\circ}$.

The line-of-sight photospheric magnetic field is provided by MDI/SOHO (Michelson Doppler Imager, Scherrer et al. 1995). As magnetograph, MDI measures the Zeeman splitting of the NiI line at $6767.8 \AA$. Figure 1 shows the distribution of the photospheric field of AR 8151 observed on February 11, 1998 at 17:36 UT with a $340^{\prime \prime} \times 300^{\prime \prime}$ field-of-view (extracted from the $96 \mathrm{~min}$ full-disk image). The spatial resolution is $2^{\prime \prime}$. AR 8151 has a near-bipolar magnetic distribution with a leading negative sunspot (black) and a diffuse positive polarity (white). The length between the two opposite polarities is estimated to $130^{\prime \prime}$. Here it is important to note that most

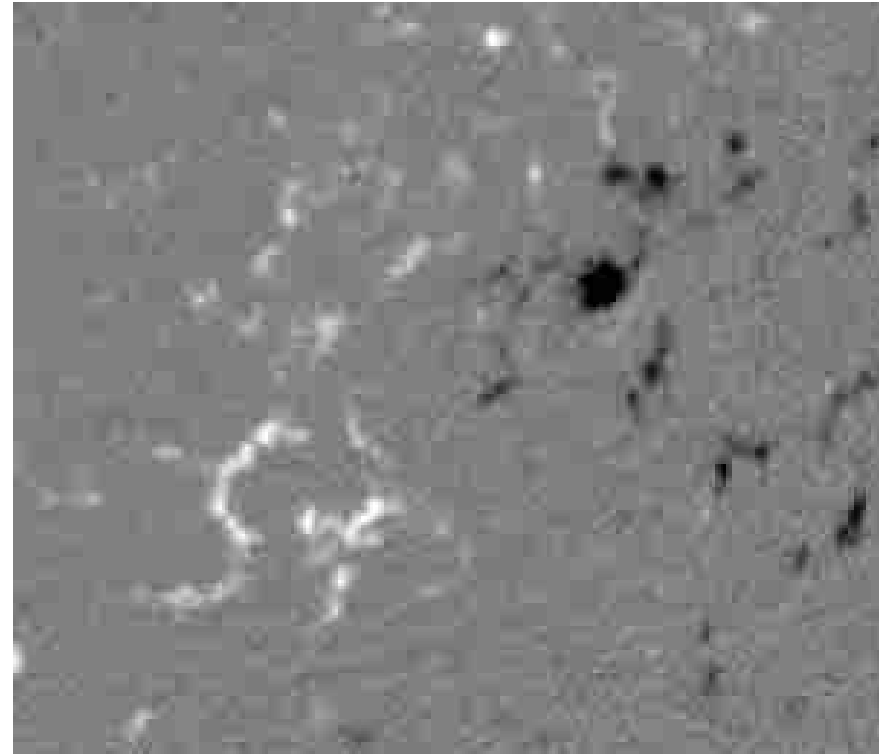

Fig. 1. Photospheric distribution of the line-of-sight magnetic field obtained by MDI/SOHO on February 11, 1998 at 17:36 UT. Black (white) pixels are negative (positive) values of the magnetic flux. The spatial resolution is $2^{\prime \prime}$. The field-of-view is $340^{\prime \prime} \times 300^{\prime \prime}$.

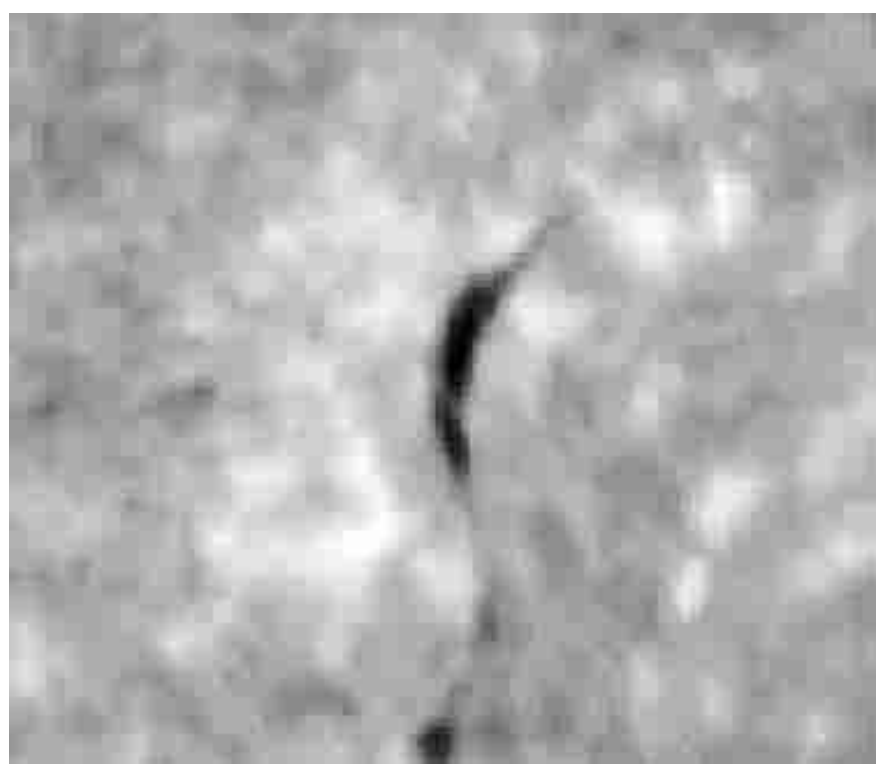

Fig. 2. $\mathrm{H} \alpha$ observation from the Paris-Meudon Observatory on February 11, 1998 at 08:58 UT. A filament is clearly visible as a dark feature aligned with the inversion line of the line-of-sight magnetic field (see Fig.1). The spatial resolution is 2.3". The field-of-view overlaps Fig. 1.

reconstructed active regions had a more compact and intense magnetic field (e.g. McClymont \& Mikic 1994). This feature will be developed in Sect. 3.

At the chromospheric and the low corona level, the structure of AR 8151 can be described by the $\mathrm{H} \alpha$ image from the Paris-Meudon Observatory on February 11, 1998 at 08:58 UT (see Fig. 2). The spatial resolution is 2.3". A filament (dark feature) is clearly observed in AR 8151. This elongated structure (core length of $90^{\prime \prime}$ ) is aligned with the inversion line of the 


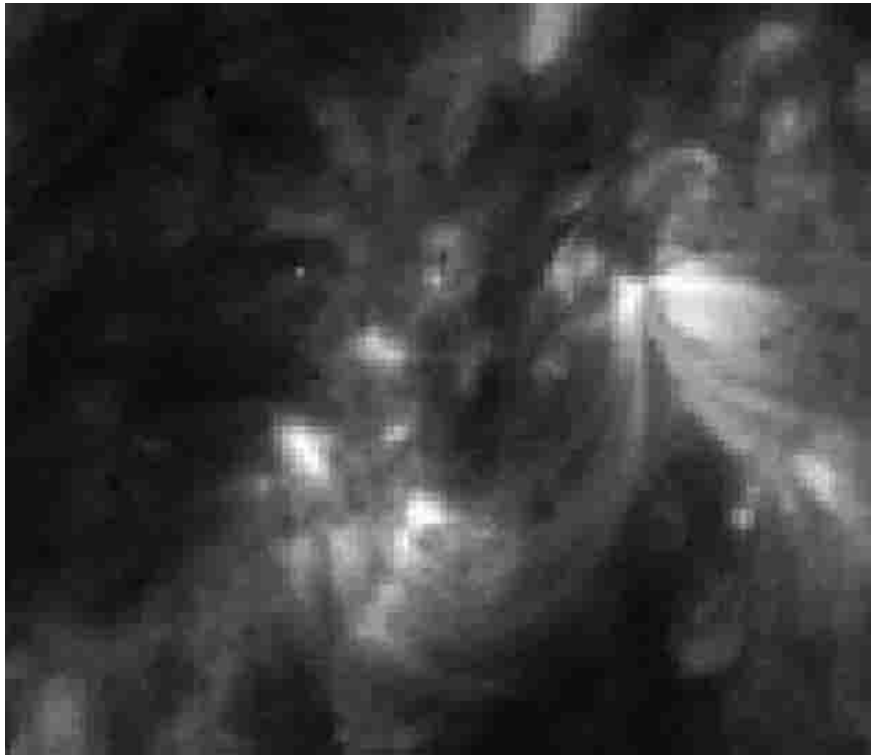

Fig. 3. EUV coronal observation from EIT/SOHO in the FeXII line at $195 \AA$ A on February 11, 1998 at 08:51 UT. A system of coronal loops is observed connecting the opposite polarities of AR 8151. The spatial resolution is $2.8^{\prime \prime}$. The field-of-view is $340^{\prime \prime} \times 300^{\prime \prime}$.

line-of-sight magnetic field (Fig. 1). The width of the filament is estimated to be $10^{\prime \prime}$.

A system of coronal loops (Fig. 3) is observed in the corona by EIT/SOHO (EUV Imaging Telescope, Delaboudinière et al. $1995)$ in the $195 \AA$ FeXII line on February 11, 1998 at 08:51 UT. The system connects the two opposite main polarities (Fig. 1). The spatial resolution is $5.2^{\prime \prime}$ with the same $340^{\prime \prime} \times 300^{\prime \prime}$ field-of-view.

The soft X-ray image of AR 8151 (Fig. 4) is provided by SXT/Yohkoh (Soft X-ray Telescope, Tsuneta et al. 1991). The spatial resolution is 2.5". Again, the field-of-view is $340^{\prime \prime} \times$ $300^{\prime \prime}$. One observes a sigmoid connecting the two polarities of AR 8151. The core of the sigmoid is aligned with the inversion line of the line-of-sight magnetic field (see Fig. 1). The forward S-shaped structure is in agreement with the chirality rules defined by Canfield \& Pevtsov (1999): in the South (resp. North) hemisphere, sigmoids have preferencially $(\sim 2 / 3)$ S-shaped (resp. inverse S-shaped) configurations.

A slow CME was both observed by EIT/SOHO and LASCO/SOHO (Régnier et al. 1999a,b). The CME was observed on February 12, 1998 at 13:51 UT with a velocity in the plane of sky estimated to $280 \mathrm{~km} \mathrm{~s}^{-1}\left( \pm 20 \mathrm{~km} \mathrm{~s}^{-1}\right)$. If we assume that the development of the CME is radial, the estimated velocity of the CME is less than $540 \mathrm{~km} \mathrm{~s}^{-1}$. A time series of EIT images at $195 \AA$ was recorded during the eruptive event with a temporal spacing of $15 \mathrm{~min}$. No EIT flare is observed before or after the CME. The magnetic evolution of AR 8151 does not reveal magnetic changes such as flux emergence.

For all those features, AR 8151 is a good candidate for our study of the existing relation between magnetic structures (filaments, sigmoids) and eruptive events (CMEs, prominence eruptions, flares).

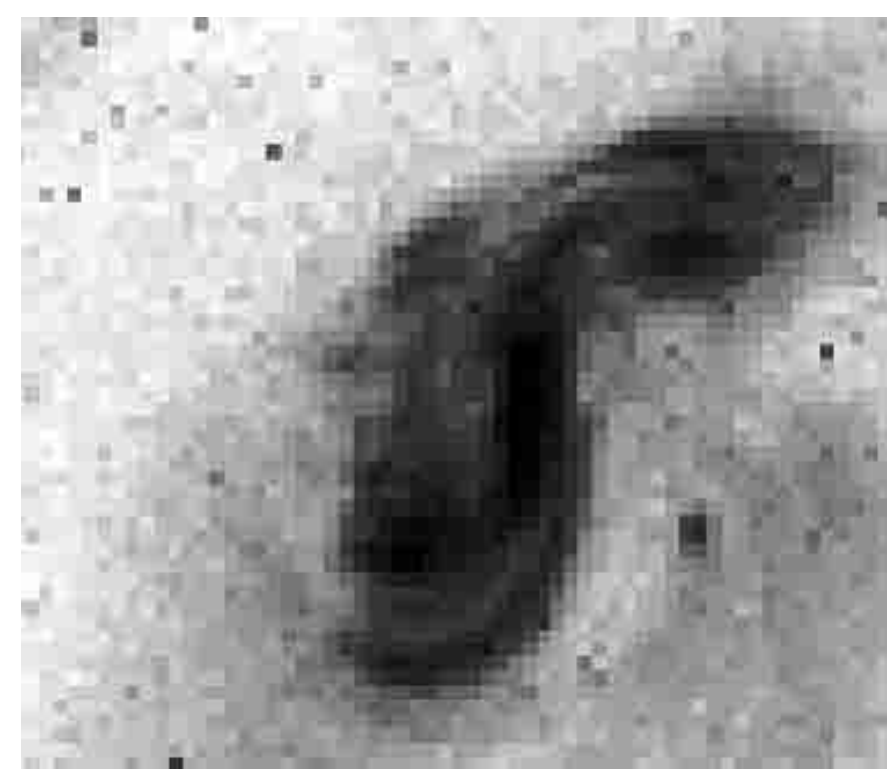

Fig. 4. Soft X-ray image from SXT/Yohkoh on February 11, 1998 at 09:19 UT (negative image). A sigmoid is observed as dark feature. The central part of the sigmoid is aligned with the inversion line of the line-of-sight magnetic field (see Fig. 1). The spatial resolution is $2.5^{\prime \prime}$. The field-of-view is $340^{\prime \prime} \times 300^{\prime \prime}$.

\section{Vector magnetic field of AR 8151}

In this section, we describe the vector magnetic field of AR 8151 and we especially focus on the measurements of transverse magnetic fields and on calculations of the current density and $\alpha(x, y, z)$ on the photosphere.

\subsection{Magnetic data}

IVM (Imaging Vector Magnetograph, Mees solar Observatory, Hawaii, Mickey et al. 1996) measures the four Stokes parameters $(I, U, Q, V)$ for the $6302.5 \AA$ FeI line on February 11, 1998 at 17:36 UT. To obtain the vector magnetic field from the Stokes parameters, the raw data are first corrected for the instrumental and the Earth's atmosphere systematic effects (see Labonte et al. 1999). The magnetic field parameters (strength, polar and azimuthal angles) are inferred from Stokes profiles using the "weak-field" approximation (Jefferies et al. 1989; Jefferies \& Mickey 1991). This approximation is valid up to a magnetic splitting of 1.5 times the Doppler width of the observed line (Jefferies \& Mickey 1991). For the $6302.5 \AA$ FeI line, the maximum magnetic splitting corresponds to a magnetic field strength of about $1500 \mathrm{G}$ (Mickey et al. 1996). The three magnetic components are $B_{\mathrm{LOS}}$ the line-of-sight component, $B_{\text {Trans }}$ and $B_{\text {Azim }}$ the two transverse components in the plane perpendicular to the line-of-sight. The equations allowing the inversion of Stokes parameters introduce a $180^{\circ}$-ambiguity on the azimuthal component $B_{\text {Azim }}$ which can be resolved using the method detailed in Canfield et al. (1993):

- in first approximation, the angle $B_{\mathrm{Azim}}$ is chosen as the closest angle to the potential field derived from $B_{\mathrm{LOS}}$; 

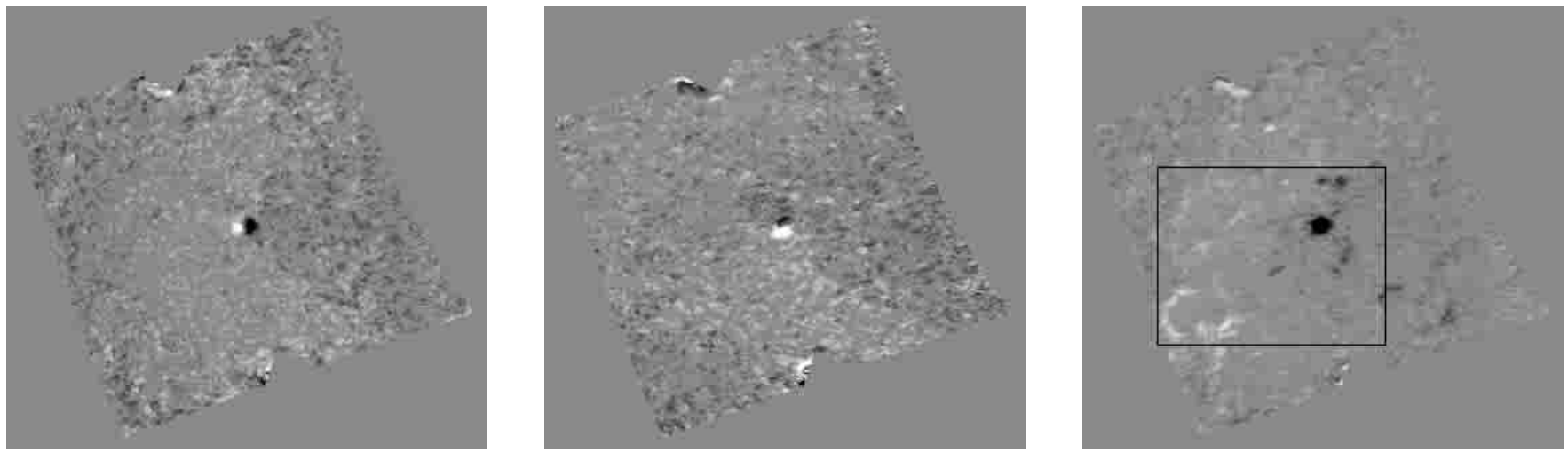

Fig. 5. The three components of the magnetic field deduced from the IVM data in heliographic coordinates: $B_{x}$ (left), $B_{y}$ (center), $B_{z}$ (right). The black box on the $B_{z}$ image is the area selected for the calculations.

Table 1. Minimal and maximal values of the three magnetic field components after the disk center heliographic transformation on the selected area of Fig. 5 (right).

\begin{tabular}{cccc}
\hline \hline & min. value & max. value & mean value \\
\hline$B_{x, \text { phot }}(\mathrm{G})$ & -755 & 885 & 18 \\
$B_{y, \text { phot }}(\mathrm{G})$ & -545 & 820 & 28 \\
$B_{z, \text { phot }}(\mathrm{G})$ & -1470 & 420 & 28 \\
$J_{z, \text { phot }}\left(\mathrm{mA} \mathrm{m}^{-2}\right)$ & -30 & 50 & 3 \\
$\alpha_{\text {phot }}\left(\mathrm{Mm}^{-1}\right)$ & -1 & 1 & see Sect. 3.2 \\
\hline
\end{tabular}

- the three components $\left(B_{\mathrm{LOS}}, B_{\text {Trans }}, B_{\text {Azim }}\right)$ are transformed into the disk center heliographic coordinates $\left(B_{x, \text { phot }}\right.$, $B_{y, \text { phot }}, B_{z \text {, phot }}$ ) (Venkatakrishnan et al. 1988);

- for current-carrying active regions, the constant- $\alpha$ forcefree field is computed and the orientation of the horizontal field is selected to give the closest match to the linear force-free field;

- the next step is to minimize the angle between neighboring vectors;

- for regions with strong magnetic field strength and a high shear, the minimization of the divergence $\nabla \cdot \boldsymbol{B}$ gives the orientation of the horizontal field;

- in regions of field strength below the noise level, the orientation is given by the minimization of the electric current.

The field-of-view is $280^{\prime \prime}$ square with a spatial resolution of $1.1^{\prime \prime}$. The time resolution is $2 \mathrm{~min}$. The three components (vertical and horizontal) are shown in Fig. 5 (see Table 1 for minimal and maximal values of the magnetic field). In order to eliminate the zero magnetic field values due to the heliographic transformation and excessively high values due to instrumental side effects, we select a $160^{\prime \prime} \times 140^{\prime \prime}$ area as defined in Fig. 5 (right).

If we compare the vertical component of the magnetic field observed by IVM (Fig. 5 right) and MDI (Fig. 1) magnetographs, we note that the IVM field-of-view does not contain some polarities surrounding the main positive and negative polarities. Therefore, we create a composite map (Fig. 6). We extend the IVM field-of-view with MDI data: the line-of-sight magnetic field from MDI is transformed into a vertical component assuming no transverse fields.

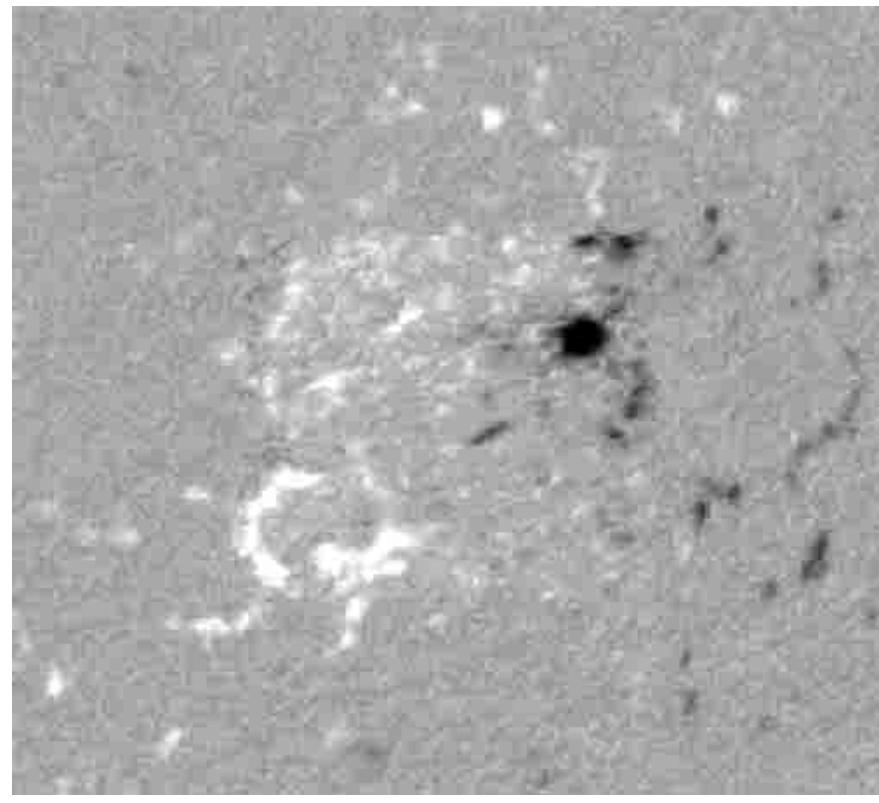

Fig. 6. Composite IVM-MDI image for the vertical component of the magnetic field $B_{z \text {, phot }}$.

For the composite image, the magnetic flux through the photospheric surface is balanced: if we consider a threshold of $50 \mathrm{G}$ on the $B_{z \text {, phot }}$ component, the magnetic fluxes for the positive $\left(\phi_{+}\right)$and negative $\left(\phi_{-}\right)$values are $\phi_{+} \sim \phi_{-} \sim 3.2 \times 10^{22} \mathrm{Mx}$ (the magnetic flux $\phi$ is given by $\phi=\sum B_{z \text {, phot }} \mathrm{d} S$ where $\mathrm{d} S$ is the pixel surface).

\subsection{The vertical current density and the force-free function $\alpha_{\text {phot }}$}

Knowing the three components of the magnetic field, we deduce the vertical current density since $\mu_{0} J_{z \text {, phot }}=$ $\left(\boldsymbol{\nabla} \wedge \boldsymbol{B}_{\text {phot }}\right)_{z}$ and the distribution of the force-free function since, $\alpha_{\text {phot }}=\frac{J_{z \text {, phot }}}{B_{z \text {, phot }}}$. In order to obtain consistent values for $J_{z \text {, phot }}$ and for $\alpha_{\text {phot }}$, we must fix thresholds (see Leka \& Skumanich 1999) on the vertical magnetic field component $\left(\left|B_{z \text {, phot }}\right|>50 \mathrm{G}\right)$ and on the transverse magnetic field components $\left(\mid B_{x}\right.$, phot $\left.|,| B_{y \text {, phot }} \mid>200 \mathrm{G}\right)$. 
The distribution of the vertical current density (see Fig. 7) exhibits strong positive and negative values in the negative magnetic polarity region $\left(\left|J_{z \text {, phot }}\right| \sim 30 \mathrm{~mA} \mathrm{~m}^{-2}\right.$, see Table 1$)$. The distribution of $J_{z}$ is quite different from usual concentrated magnetic field (and electric current density) used to reconstruct the coronal magnetic field. In Sect. 5, we will see that this kind of real data can give the $3 \mathrm{D}$ coronal magnetic configuration. The values of $\alpha_{\text {phot }}$ range between $-1 \mathrm{Mm}^{-1}$ and $1 \mathrm{Mm}^{-1}$. The mean value of $\alpha_{\text {phot }}$ for the whole active region is $\sim 0.12 \mathrm{Mm}^{-1}$. This positive mean value and the South location of AR 8151 are in agreement with the statistical study on the sign of $\alpha_{\text {phot }}$ performed by Pevtsov et al. (1995) which demonstrated that $\sim 70 \%$ of active regions observed in the South have a positive value of $\alpha_{\text {phot }}$ (see also Longcope et al. 1998). The mean values of each polarity are $0.18 \mathrm{Mm}^{-1}$ for the diffuse positive polarity and $2.18 \times 10^{-3} \mathrm{Mm}^{-1}$ for the negative polarity. The small mean value of $\alpha_{\text {phot }}$ in the negative spot hides strong positive and negative values.

The positive and negative values for the distribution of the vertical current density and of $\alpha_{\text {phot }}$ characterize the existence of return currents (since $J_{z}=\alpha B_{z}$ ) which would create in almost physical MHD process (Régnier \& Amari 2001).

\section{Reconstruction method}

Let us recall the equations governing the nlff magnetic field in the half-space $\Omega$ above the photosphere $\partial \Omega$ :

$$
\begin{aligned}
& \boldsymbol{\nabla} \wedge \boldsymbol{B}=\alpha \boldsymbol{B}, \\
& \boldsymbol{B} \cdot \boldsymbol{\nabla} \alpha=0, \\
& \nabla \cdot \boldsymbol{B}=0 .
\end{aligned}
$$

In these equations, $\alpha$ is a function of the position. In cartesian coordinates, we can write $\alpha=\alpha(x, y, z)$. Equation (1) means that the electric current density $\boldsymbol{J}\left(\mu_{0} \boldsymbol{J}=\boldsymbol{\nabla} \wedge \boldsymbol{B}\right)$ is collinear to the magnetic field $\boldsymbol{B}$. From Eq. (2) (divergence of Eq. (1)), $\alpha$ is constant along each field line. The set of Eqs. (1)-(3) is completed by boundary conditions on the photosphere $\partial \Omega$ and it is commonly assumed that the magnetic field strength vanishes at infinity:

$$
\lim _{|\boldsymbol{r}| \rightarrow \infty}|\boldsymbol{B}|=0 .
$$

The stongly nonlinear system of equations (Eqs. (1)-(3)) can be approached by the sequence of linear problems (Grad \& Rubin 1958; Aly 1989):

$$
\begin{aligned}
& \boldsymbol{B}^{(n)} \cdot \boldsymbol{\nabla} \alpha^{(n)}=0 \quad \text { in } \Omega, \\
& \left.\alpha^{(n)}\right|_{\partial \Omega^{+}}=h,
\end{aligned}
$$

and

$$
\begin{aligned}
& \boldsymbol{\nabla} \wedge \boldsymbol{B}^{(n+1)}=\alpha^{(n)} \boldsymbol{B}^{(n)} \text { in } \Omega, \\
& \boldsymbol{\nabla} \cdot \boldsymbol{B}^{(n+1)}=0 \quad \text { in } \Omega, \\
& \left.B_{z}^{(n+1)}\right|_{\partial \Omega}=g, \\
& \lim _{|r| \rightarrow \infty}|\boldsymbol{B}|=0 .
\end{aligned}
$$

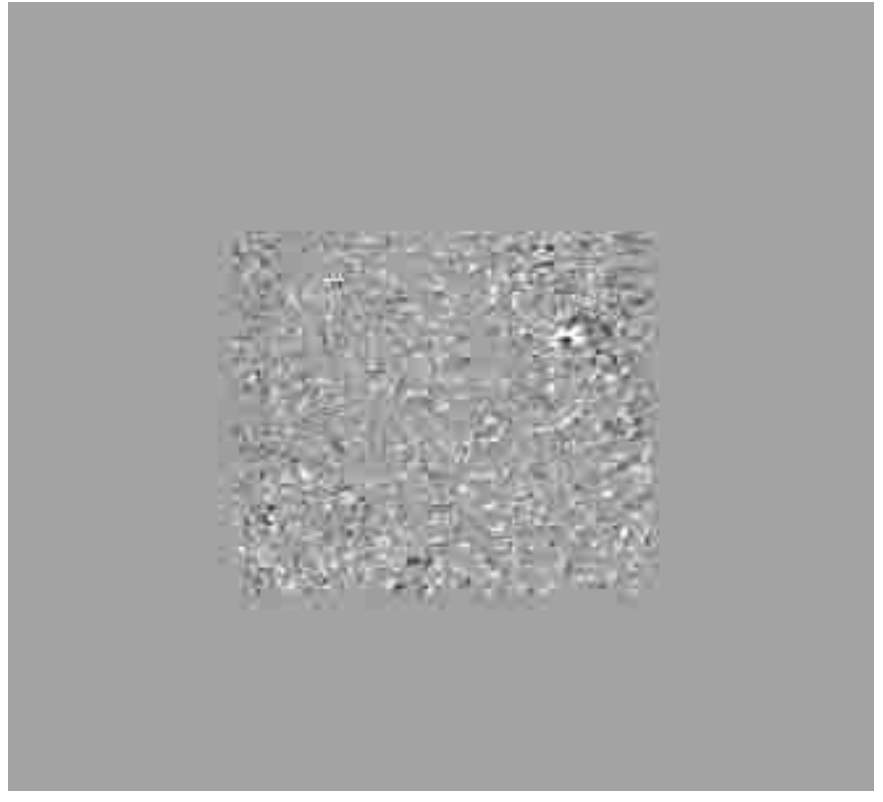

Fig. 7. Distribution of the vertical current density $J_{z \text {, phot }}$ on the photosphere deduced from the composite magnetic field map. $J_{z \text {, phot }}$ is equal to zero where the MDI data has been added.

Equations (5) correspond to the transport of $\alpha$ along field lines. The boundary condition $h$ is given by the photospheric distribution of $\alpha$ on $\partial \Omega^{+}$(the part of $\partial \Omega$ where $B_{z}>0$ ). One can also choose the part of $\partial \Omega$ where $B_{z}<0$, i.e. $\partial \Omega^{-}$. In general, the polarity in which the magnetic flux is more accurate (e.g. in the sunspot) allows us to determine the domain from which we transport $\alpha$ along field lines. The system of Eq. (6) determines the magnetic field $\boldsymbol{B}^{(n+1)}$ in $\Omega$ from the electric current density $\alpha^{(n)} \boldsymbol{B}^{(n)}$ using the observed photospheric vertical magnetic field $g$ as boundary condition on $\partial \Omega$. Starting from the potential field $\left(\boldsymbol{B}^{(0)}=\boldsymbol{B}_{0}\right)$, one solves the system (6) and the system (5).

A method has been developed by Amari et al. (1997) (see also Amari et al. 1999) using the vector potential representation in order to guarantee Eq. (3):

$$
\boldsymbol{B}=\nabla \wedge \boldsymbol{A} \text { in } \Omega
$$

The vector potential $\boldsymbol{A}$ is unique for the following gauge conditions (Amari et al. 1997; Amari et al. 1999):

$\nabla \cdot \boldsymbol{A}=0$ in $\Omega$

$\nabla_{\mathrm{t}} \cdot \boldsymbol{A}_{\mathbf{t}}=0 \quad$ on $\partial \Omega$

where the subscript $t$ means the trace of the operator or the vector on the boundary $\partial \Omega$. This boundary gauge condition is determined from the vertical photospheric magnetic field $g$ (see Eq. (6)) using the scalar potential $\chi$ :

$\boldsymbol{A}_{\mathbf{t}}=\nabla^{\perp} \chi \quad$ on $\partial \Omega$

where $\chi$ is the solution of:

$$
\begin{aligned}
& -\Delta_{2} \chi=g \text { on } \partial \Omega, \\
& \chi=0 \text { or } \partial_{n} \chi=0 \text { on } \Gamma,
\end{aligned}
$$


where $\Gamma$ is the border of $\partial \Omega$ and $\Delta_{2}$ is the Laplacian operator on $\partial \Omega$. The gauge condition in $\Omega$ implies that the system (6) can be rewritten as follows:

$$
\begin{aligned}
& -\Delta \boldsymbol{A}^{(n+1)}=\alpha^{(n)} \nabla \wedge \boldsymbol{A}^{(n)} \quad \text { in } \Omega, \\
& \boldsymbol{A}_{t}^{(n+1)}=\nabla^{\perp} \chi \quad \text { on } \partial \Omega, \\
& \partial_{n} \boldsymbol{A}_{n}^{(n+1)}=0 \quad \text { on } \partial \Omega .
\end{aligned}
$$

This method, so-called the vector potential Grad-Rubin-like method, is the basis of the numerical code XTRAPOL dedicated to the reconstruction of coronal magnetic fields as forcefree fields. In practice, we use the potential field $\boldsymbol{B}_{0}$ (no electric current, $\alpha=0$ ) as the initial equilibrium state. We progressively inject the distribution of $\alpha$ deduced from vector magnetograms ( $N$ injections). For each value of $N$, we solve the systems (13) and (5) for a finite value of $n$ (number of Grad-Rubin iterations) to obtain a new equilibrium state. To compute the nlff forcefree field, the domain $\Omega$ is a finite size box. In order to satisfy Eq. (4), we must determine a boundary condition on each side wall of the box other than the side associated with the photosphere. Therefore we impose that the normal component of the magnetic field vanishes on each side of the box:

$B_{n}=0 \quad$ on $\Sigma-\partial \Omega$

where $\Sigma$ is the surface of the finite size box $\Omega$. This boundary condition imposes that the photospheric magnetic flux be balanced (already imposed by Eq. (3)) and that the observed active region be magnetically disconnected from other active regions. To reduce the computation time we use a non-uniform 3D grid characterized by a continuous evolution in each direction. The smallest grid step is limited by the spatial resolution of the magnetograph.

\section{Non-linear force-free field of AR 8151}

\subsection{The computed magnetic field}

As mentioned in the preceding Section, the method to reconstruct the 3D coronal magnetic field uses the vertical component of the magnetic field $B_{z}$ (i.e. $g$ in Eq. (6)) and the distribution of $\alpha$ (i.e. $h$ in Eq. (5)) for one polarity on the pho-

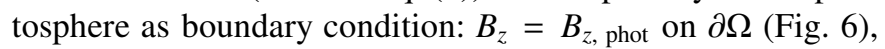
$\alpha=\alpha_{\text {phot }}$ on $\partial \Omega^{-}$(Fig. 8). $\alpha$ is transported from $\partial \Omega^{-}$which corresponds to the sunspot magnetic field. The continuous nonuniform grid takes into account the photospheric variation of the magnetic flux: the grip steps are shorter where the magnetic flux is higher.

The three components of the magnetic field $\left(B_{x}, B_{y}, B_{z}\right)$ are computed in a box of $148 \times 128 \times 80$ grid steps. These components satisfy the equations mentioned in the preceding section. To visualize the magnetic configuration of AR 8151, we plot the magnetic field lines defined as follows:

$\frac{\mathrm{d} x}{B_{x}}=\frac{\mathrm{d} y}{B_{y}}=\frac{\mathrm{d} z}{B_{z}}$.

Field lines fill the entire volume $\Omega$. We have chosen to study the three relevant flux tubes defined in Fig. 9. We investigate the

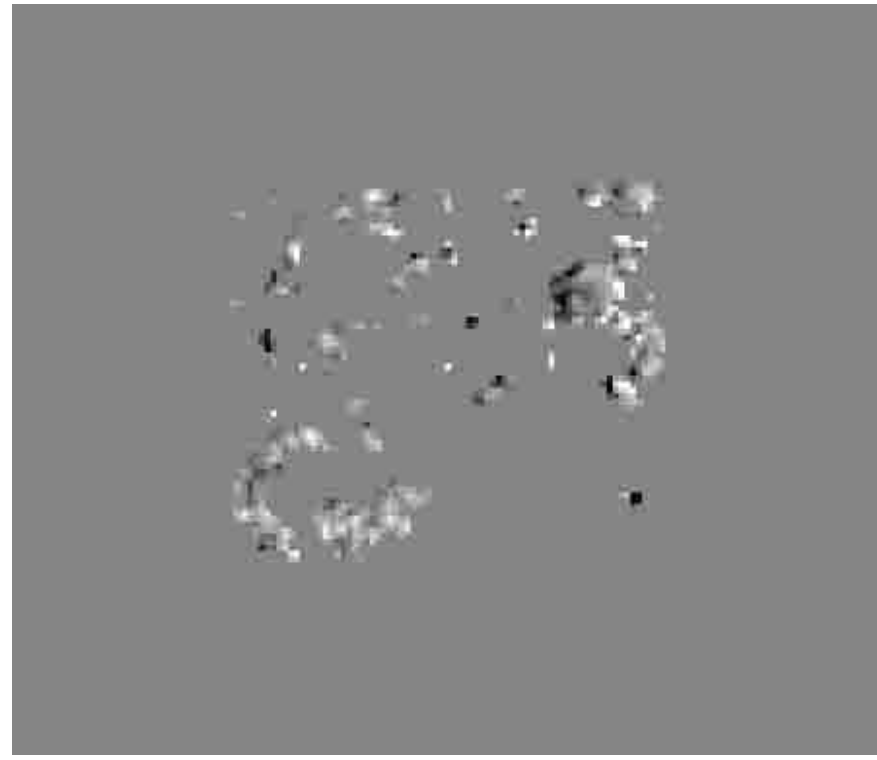

Fig. 8. Distribution of the force-free function $\alpha$ on the photosphere. Each polarity has mixed signs of $\alpha_{\text {phot }}$ which may account for return currents. (Black (resp. white) pixels represent negative (resp. positive) values of $\alpha_{\text {phot }}$ )

topological (twist and shear) and geometrical features (height, width) of these three flux tubes:

(1) the flux tube has strong twist ( $1-1.2$ turns). The value of the vertical magnetic field is estimated to $200 \mathrm{G}$. The value of $\alpha$ is positive and consequently the electric current density has the same direction than the magnetic field. The height of the flux tube is estimated to be $60 \mathrm{Mm}$ and the width to be $10 \mathrm{Mm}$;

(2) the flux tube is sheared and moderately twisted ( 0.5-0.6 turns). The vertical magnetic field on the photosphere is estimated to $70 \mathrm{G}$. As $\alpha$ has a negative value, the current density has the opposite direction to the magnetic field. The height of the flux tube is estimated to be $40 \mathrm{Mm}$ and its width to be $5 \mathrm{Mm}$;

(3) the flux tube is a quasi-potential flux tube (no twist) but the shear is high (we define a quasi-potential flux tube as a potential-like arcade which is not perpendicular to the associated magnetic inversion line of the longitudinal field). The photospheric vertical magnetic field is estimated to $150 \mathrm{G}$. The current density has the opposite direction to the magnetic field $(\alpha<0)$. The height of (3) is estimated to be $60 \mathrm{Mm}$ and its width to be $15 \mathrm{Mm}$.

(The shear is defined as the angle $\psi$ between the flux tube and the photospheric magnetic field inversion line. If $\psi \sim 0[\pi]$, the flux tube is highly sheared.) The reconstruction method applied for AR 8151 is able to provide magnetic flux tubes with various height scales and different topology determining by the wide range of electric current density.

Note the existence of twisted flux tubes on the magnetic configuration. Hood \& Priest (1981) have demonstrated that a twisted flux tube (assuming cylindrical geometry and considering the line-tying effect) is stable when the twist $\Phi$ is less than one turn whatever geometrical parameter such as length, width 

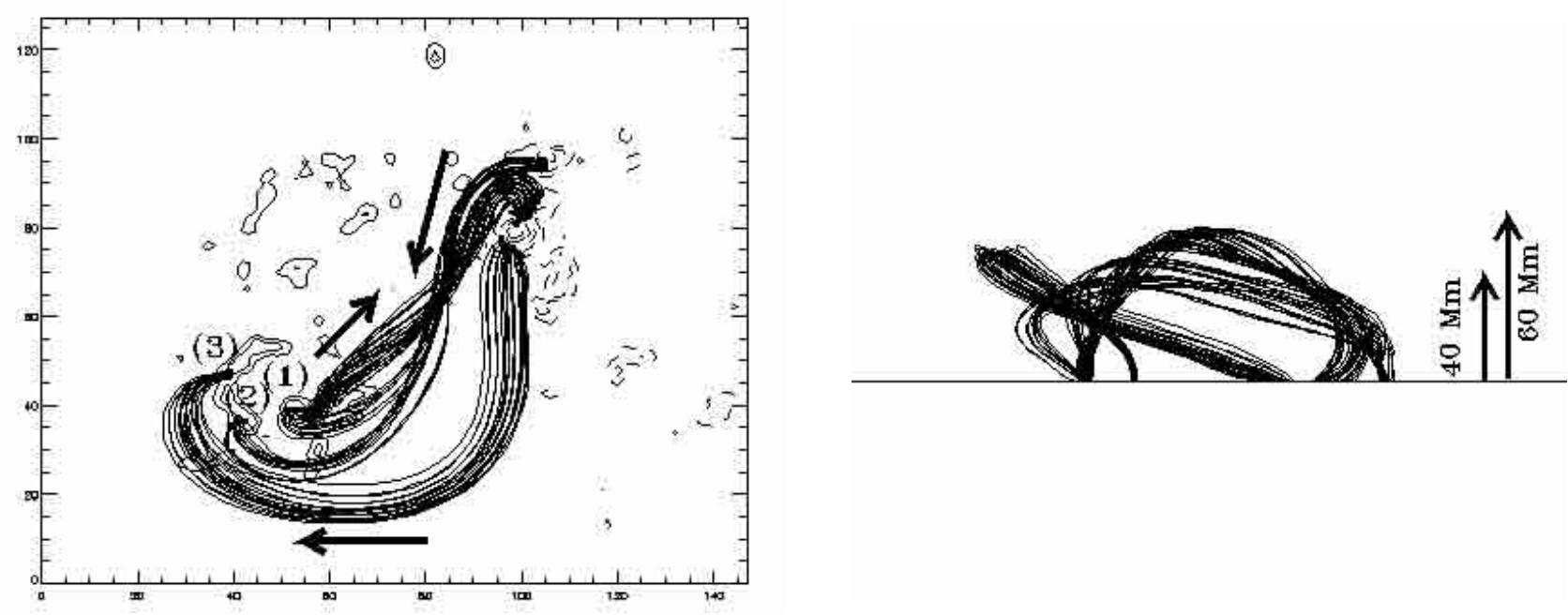

Fig. 9. Three characteristic magnetic flux tubes seen from top view (left) and from side view (right). The photospheric positive (resp. negative) polarity is drawn as solid (resp. dashed) contours. Black arrows (left) indicate the direction of the electric current density on each flux tube. The estimated height of flux tubes is indicated on the right image.

and height (see also Baty 2001 for a review of recent studies of twisted flux tube kink instability and Amari \& Luciani 1999 for 3D MHD disruption of a twisted flux tube). The value of $\Phi_{\text {crit }}$ at which the twisted flux tube becomes unstable mainly depends on the height and on the width of the flux tube. For flux tube (1), we estimate $\Phi_{\text {crit }} \sim 3 \pi$ or 1.5 turns. For the nlff configuration of AR 8151, the twisted flux (2) is stable and the twist of flux tube (1) is close to $\Phi_{\text {crit }}$ (but less than $\Phi_{\text {crit }}$ ). This important point for CME mechanisms is also a main point for the validity of the computed configuration: the reconstruction method is based on the finding of an equilibrium state, therefore for twisted flux tubes we should always satisfy the condition $\Phi<\Phi_{\text {crit }}$.

\subsection{Comparison with the observations}

We now compare the nlff magnetic field lines to the coronal observations. The three characteristic flux tubes (see Fig. 9) are in good agreement with the coronal observations:

- the quasi-potential flux tube (3) matches the system of coronal loops observed by EIT at $195 \AA$ (Fig. 3) with a temperature range around $1.5 \mathrm{MK}$;

- the twisted flux tubes (1) and (2) are in agreement with the sigmoid observed by SXT (Fig. 4) with a temperature range around $2 \mathrm{MK}$. The sigmoidal shape can be described by the flux tube (2) with a negative value of $\alpha$ and by the flux tube (1) with a positive value of $\alpha$.

As mentioned in the previous section, the nlff reconstruction allows to determine the magnetic configuration of flux tubes with different heights and different values of electric currents. This is the reason why both $195 \AA$ and soft X-ray observations can be compared simultaneously with the computed nlff magnetic field. Note that the soft X-ray S-shaped structure and the EUV system of coronal loops have a typical height of $50 \mathrm{Mm}$. A thermodynamical study of each flux tube will be useful to understand the EUV and the soft X-ray enhancements. Otherwise photospheric vector magnetic observations before and after will allow us to determine the time evolution and the formation of the twisted flux tube.

\subsection{Magnetic energy $E_{m}$}

The magnetic energy $E_{\mathrm{m}}$ is defined by:

$E_{\mathrm{m}}=\int_{\Omega} \frac{B^{2}}{2 \mu_{0}} \mathrm{~d} V$.

During the largest solar flares, the magnetic energy releases is estimated to be $10^{32} \mathrm{erg}$ (Priest 1981). This magnetic energy is a small part of the magnetic energy stored in the related active region $\left(\sim 10^{34} \mathrm{erg}\right)$. Therefore, the relevant magnetic energy is the free magnetic energy: the magnetic energy which could released during an eruptive event.

For a given photospheric magnetic field, the minimum magnetic energy is obtained for the potential field (e.g. Aly 1989). The free magnetic energy of the $n l f f$ force-free field has a maximum value $\Delta E_{\mathrm{m}}$ :

$\Delta E_{\mathrm{m}}=E_{\mathrm{m}}^{n l f f}-E_{\mathrm{m}}^{\mathrm{pot}}$

where $E_{\mathrm{m}}^{\mathrm{nlff}}$ (resp. $E_{\mathrm{m}}^{\mathrm{pot}}$ ) is the magnetic energy of the nlff (resp. potential) configuration given by Eq. (16). According to the Taylor's hypothesis (Taylor 1974), the minimum magnetic energy for a given magnetic helicity is obtained for a constant- $\alpha$ force-free field. In a more general case, the magnetic energy released during an eruptive event is less than expected in the Taylor's hypothesis (see Amari \& Luciani 2000; Bleybel et al. 2002). Therefore, $\Delta E_{\mathrm{m}}$ overestimates the free magnetic energy available to trigger an eruptive event. Following the Aly-Sturrock's conjecture (Aly 1991; Sturrock 1991), the "open field" magnetic energy, $E_{\mathrm{m}}^{\text {open }}$, is an upper limit of the magnetic energy. Before an eruptive event, $E_{\mathrm{m}}^{\mathrm{open}}$ can be estimated to $2 E_{\mathrm{m}}^{\mathrm{pot}}$ (see Amari et al. 2000).

For AR 8151, the magnetic energy of the potential field (initial state of the configuration) is:

$E_{\mathrm{m}}^{\mathrm{pot}}=3.8 \times 10^{31} \mathrm{erg}$. 
The magnetic energy of the nlff field is:

$E_{\mathrm{m}}^{\text {nlff }}=6.4 \times 10^{31} \mathrm{erg}$,

and the magnetic energy associated to the open field is estimated to:

$E_{\mathrm{m}}^{\mathrm{open}}=7.6 \times 10^{31} \mathrm{erg}$.

The maximum magnetic energy released during an eruptive event in AR 8151 is estimated to:

$\Delta E_{\mathrm{m}}=2.6 \times 10^{31} \mathrm{erg}$.

The small value of $\Delta E_{\mathrm{m}}$ cannot produce a large flare and certainly explains why no EIT flare is observed. As shown by Amari \& Luciani (1999), a confined eruption of a twisted flux tube can be triggered with less than $30 \%$ of the maximum free energy. Hence with the relatively small value of the free magnetic energy and with the existence of highly twisted flux tube, the mechanism of the eruption in AR 8151 is more probably linked to the destabilization of the twisted flux tubes.

\subsection{Relative magnetic helicity $\Delta H_{m}$}

The magnetic helicity $H_{\mathrm{m}}$ takes into account the complexity of the magnetic field topology such as twist or writhe (see review by Berger 1999):

$H_{\mathrm{m}}=\int_{\Omega} \boldsymbol{A} \cdot \boldsymbol{B} \mathrm{d} V$

where $\boldsymbol{A}$ is the vector potential associated to the vector magnetic field $\boldsymbol{B}$. With this definition, $H_{\mathrm{m}}$ depends on the gauge condition imposed on $\boldsymbol{A}$. To overcome this dependancy, Berger \& Field (1984) defined a new quantity named the relative magnetic helicity $\Delta H_{\mathrm{m}}$ :

$$
\begin{aligned}
\Delta H_{\mathrm{m}}= & \int_{\Omega}\left(\boldsymbol{A}-\boldsymbol{A}_{0}\right) \cdot\left(\boldsymbol{B}+\boldsymbol{B}_{0}\right) \mathrm{d} V \\
& +\int_{\partial \Omega} \chi\left(\boldsymbol{B}+\boldsymbol{B}_{0}\right) \cdot \boldsymbol{n} \mathrm{d} S
\end{aligned}
$$

where $\boldsymbol{A}_{0}$ is the vector potential associated to the potential magnetic field $\boldsymbol{B}_{0}, \partial \Omega$ is the surface bounding the volume $\Omega$ and $\boldsymbol{n}$ is the normal to $\partial \Omega$. $\chi$ depends on $\boldsymbol{B}$ and $\boldsymbol{B}_{0}$ (Berger \& Field 1984). If $\Omega$ is the half-space above the photosphere, the surface integral on $\partial \Omega$ tends to zero. If the normal component of the magnetic field vanishes on $\partial \Omega$, the surface integral is strictly equal to zero. To compute the $n l f f$ field, we impose that the normal component of the magnetic field vanishes on the surface $\Sigma-\partial \Omega$ (see Eq. (14)) and that the boundary flux on $\partial \Omega$ vanishes. Therefore, $\chi$ is equal to zero for simply connected domains (Low 1999). For our boundary conditions, the relative magnetic helicity is given by:

$\Delta H_{\mathrm{m}}=\int_{\Omega}\left(\boldsymbol{A}-\boldsymbol{A}_{0}\right) \cdot\left(\boldsymbol{B}+\boldsymbol{B}_{0}\right) \mathrm{d} V$.

In the case of AR 8151, the relative magnetic helicity for the nlff magnetic configuration is:

$\Delta H_{\mathrm{m}}^{n l f f}=4.7 \times 10^{34} M x^{2}$.
(Obviously the relative magnetic helicity is equal to zero for the potential field.) The value of $\Delta H_{\mathrm{m}}$ is positive, as is the mean value of $\alpha$, which indicates that the magnetic helicity and the force-free parameter have the same chirality rules (Berger \& Ruzmaikin 2000). This value of the relative magnetic helicity cannot give relevant information of the CME's mechanisms. The appropriate parameter to study the dynamics of eruption is the time evolution of magnetic helicity, which is not investigated in this paper.

\section{Discussion and conclusions}

We have detailed a method to obtain the $3 \mathrm{D}$ coronal magnetic field of an active region using photospheric vector magnetograms as boundary conditions. This method comprises into three main steps:

- the analysis of the magnetic data including the consistency of the transverse magnetic field, the resolution of the $180^{\circ}$ ambiguity, the computation of the vertical current density and of the force-free function on the photosphere;

- the reconstruction of the non-constant- $\alpha$ force-free field based on the vector potential Grad-Rubin-like method. The boundary condition on the photosphere is given by the vertical component of the magnetic field and by the distribution of $\alpha$ for one polarity;

- the visualization of the nlff field and the derivation of relevant quantities as the free magnetic energy and the relative magnetic helicity.

We analysed the 3D magnetic configuration for the AR 8151 chosen for exhibiting a filament, a sigmoid and a system of coronal loops and for being the site of a slow CME. The reconstructed coronal magnetic field is in agreement with observations provided by EIT/SOHO and SXT/Yohkoh. In particular, we associate the observed sigmoid with twisted flux tubes. The filament cannot be directly reconstructed due to its low magnetic field strength on the photosphere (e.g. Rust 1970) and due to the fixed threshold defined by the noise level (see Sect. 3). For AR 8151, two relevant parameters are derived: the maximum free magnetic energy of the entire configuration $\Delta E_{\mathrm{m}}=2.6 \times 10^{31} \mathrm{erg}(\sim 45 \%$ over the magnetic energy of the potential field), the relative magnetic helicity $\Delta H_{\mathrm{m}}=4.7 \times 10^{34} \mathrm{Mx}^{2}$.

Determining the $3 \mathrm{D}$ magnetic configuration at a given time before the eruption affords us the ability to evaluate the validity of the different mechanisms of CME. Most CME mechanisms involve magnetic reconnection in the corona. Three main classes of CMEs are often discussed: the "breakout" model (Antiochos et al. 1999), the twisted flux rope model (Amari et al. 2000) and the tether-cutting model (Sturrock 1989). For the breakout model, the reconnection of arcades occurs above an active region with a complex topology. The instability of the twisted flux rope creates a current sheet implying reconnection in the corona. The tether-cutting model assumes that a flux rope overlaid by arcades rises in the corona and current sheets are formed below the flux rope. The latter two models do not required complex topology on the photophere. For AR 8151, the distribution of the vertical magnetic field on the photosphere is 
dipolar (see Fig. 1). The reconstructed 3D magnetic field evidences twisted magnetic flux tubes close to the instability condition (see Sect. 5). Hence, the breakout model seems not to be the scenario of AR 8151's CME. Most probably the destabilization of twisted flux tubes and/or the formation of current sheets under the rising twisted flux tubes can explain the existence of the CME.

As the snapshot of the 3D magnetic configuration is given $\sim 20$ hours before the eruption, we cannot expect that the twisted flux rope model is the unique mechanism to trigger the CME. To clarify the CME mechanism, we propose to use a time series of vector magnetic fields in order to follow the evolution of the nlff field topology before and after eruptive events. This time evolution will also give information on the storage of magnetic energy and on the conservation of relative magnetic helicity. As the filament cannot be directly reconstructed, we extend our method to the finding of magnetic dips which support filament material (see Régnier 2001). Thermodynamic aspects of isolated field lines are currently being investigated. In a further paper, we compare the topological and geometrical configurations of the current-free, the lff and the nlff magnetic field configurations and we investigate support and modeling of prominences in such configurations.

Acknowledgements. We thank D. L. Mickey and B. J. LaBonte for providing IVM data. We are grateful to R. C. Canfield and R. J. Leamon for comments on a previous version of this article. We wish to thank the Centre National d'Études Spatiales (CNES) for its financial support. Data used here from Mees Solar Observatory, University of Hawaii, are produced with the support of NASA grant NAG 5-4941 and NASA contract NAS8-40801. Data processing of SOHO and Yohkoh images was performed using the facilities of the MEDOC archive center (Institut d'Astrophysique Spatiale, Orsay, France).

\section{References}

Aly, J. J. 1989, Sol. Phys., 120, 19

Aly, J. J. 1991, ApJ, 375, L61

Amari, T., \& Démoulin, P. 1992, in Méthodes de détermination des champs magnétiques solaires et stellaires, GdR Magnétisme dans les étoiles de type solaire, 187

Amari, T., Aly, J. J., Luciani, J. F., Boulmezaoud, T. Z., \& Mikic, Z. 1997, Sol. Phys., 174, 129

Amari, T., Boulmezaoud, T. Z., \& Mikic, Z. 1999, A\&A, 350, 1051

Amari, T., \& Luciani, J. F. 1999, ApJ, 515, L81

Amari, T., \& Luciani, J. F. 2000, Phys. Rev. Lett., 84, 1196

Amari, T., Luciani, J. F., Mikic, Z., \& Linker, J. 2000, ApJ, 529, L49

Antiochos, S. K., Devore, C. R., \& Klimchuk, J. A. 1999, ApJ, 510, 485

Aschwanden, M. J., Newmark, J. S., Delaboudinière, J. P., et al. 1999a, ApJ, 515, 842

Aschwanden, M. J., Kosugi, T., Hanaoka, Y., Nishio, M., \& Melrose, D. B. 1999b, ApJ, 526, 1026

Aschwanden, M. J., Alexander, D., Hurlburt, N., et al. 2000, ApJ, 531, 1129

Baty, H. 2001, A\&A, 367, 321

Berger, M. A., \& Field, G. B. 1984, Journal of Fluid Mechanics, 147, 133

Berger, M. A., 1999, in Magnetic Helicity in Space and Laboratory Plasmas, ed. M. R. Brown, R. C. Canfield, \& A. A. Pevtsov, Geophysical Monograph 111, AGU, 1
Berger, M. A., \& Ruzmaikin, A. 2000, J. Geophys. Res., 105, 10481

Bleybel, A., Amari, T., van Driel-Gesztelyi, L., \& Leka, K. D. 2002, submitted

Brosius, J. W., Willson, R. F., Holman, G. D., \& Schmelz, J. T. 1992, ApJ, 386, 347

Canfield, R. C., de la Beaujardière, J. F., Fan, Y., et al. 1993, ApJ, 411, 362

Canfield, R. C., \& Pevtsov, A. A. 1999, in Magnetic Helicity in Space and Laboratory Plasmas, ed. M. R. Brown, R. C. Canfield, \& A. A. Pevtsov, Geophysical Monograph 111, AGU, 197

Canfield, R. C., Hudson, H. S., \& Pevtsov, A. A. 2000, IEEE Trans. Plasma Sci., 28, 1786

Delaboudinière, J.-P., Artzner, G. E., Brunaud, J., et al. 1995, Sol. Phys., 162, 291

Démoulin, P., Henoux, J. C., Mandrini, C. H., \& Priest, E. R. 1997, Sol. Phys., 174, 73

Gold, T., \& Hoyle, F. 1960, MNRAS, 120, 89

Grad, H., \& Rubin, H. 1958, Proc. 2nd Int. Conf. on Peaceful Uses of Atomic Energy, vol. 31, Geneva, United Nations, 190

Hood, A. W., \& Priest, E. R. 1981, Geophys. Astrophys. Fluid Dynamics, 17, 297

Jefferies, J. T., Lites, B. W., \& Skumanich, A. 1989, ApJ, 343, 920

Jefferies, J. T., \& Mickey, D. L. 1991, ApJ, 372, 694

Labonte, B. J., Mickey, D. L., \& Leka, K. D. 1999, Sol. Phys., 189, 1

Leka, K. D., \& Skumanich, A. 1999, Sol. Phys., 188, 3

Lin, H., Penn, M. J., \& Tomczyk, S. 2000, ApJ, 541, L83

Longcope, D. W., Fisher, G. H., \& Pevtsov, A. A. 1998, ApJ, 507, 417

Low, B. C. 1999, in Magnetic Helicity in Space and Laboratory Plasmas, ed. M. R. Brown, R. C. Canfield, \& A. A. Pevtsov, Geophysical Monograph 111, AGU, 25

Mandrini, C. H, Démoulin, P., Bagala, L. G., et al. 1997, Sol. Phys., 174, 229

Mickey, D., Canfield, R. C., LaBonte, B. J., et al. 1996, Sol. Phys, 168, 229

McClymont, A. N., \& Mikic, Z. 1994, ApJ, 422, 899

McClymont, A. N., Jiao, L., \& Mikic, Z. 1997, Sol. Phys., 174, 191

Pevtsov, A. A., \& Canfield, R. C. 1999, in Magnetic Helicity in Space and Laboratory Plasmas, ed. M. R. Brown, R. C. Canfield, \& A. A. Pevtsov, Geophysical Monograph 111, AGU, 103

Pevtsov, A. A., Canfield, R. C., \& Metcalf, T. R. 1995, ApJ, 440, 109

Priest, E. R. 1981, in Solar Flare Magnetohydrodynamics, 1

Priest, E. R. 1984, in Solar magneto-hydrodynamics, Geophysics and Astrophysics Monographs (Dordrecht: Reidel)

Raouafi, N.-E. 2000, Ph.D. Thesis

Régnier, S. 2001, Ph.D. Thesis

Régnier, S., \& Amari, T. 2001, IAU Symp., 203, 441

Régnier, S., Amari, T., Solomon, J., Vial, J.-C., \& Mickey, D. 1999a, 8th SOHO workshop, ESA SP-446, 571

Régnier, S., Solomon, J., Vial, J.-C., Amari, T., \& Mickey, D. 1999b, 9th European Meet. Sol. Phys., ESA SP-448, 519

Rust, D. M. 1970, ApJ, 160, 315

Sakurai, T. 1981, Sol. Phys., 69, 343

Sakurai, T. 1989, Space Sci. Rev., 51, 11

Scherrer, P. H., Bogart, R. S., Bush, R. I., et al. 1995, Sol. Phys., 162, 129

Sturrock, P. A. 1989, Sol. Phys., 121, 387

Sturrock, P. A. 1991, ApJ, 380, 655

Subramanian, P., \& Dere, K. P. 2001, ApJ, 561, 372

Taylor, J. B. 1974, Phys. Rev. Lett., 33, 1139

Tsuneta, S., Acton, L., Bruner, M., et al. 1991, Sol. Phys., 136, 37

Venkatakrishnan, P., Hagyard, M. J., \& Hathaway, D. H. 1988, Sol. Phys., 115, 125

Wu, S. T., Sun, M. T., Chang, H. M., Hagyard, M. J., \& Gary, G. A. 1990, ApJ, 362, 698 